\title{
Numerical analysis of a high-resolution fast tunable filter based on an intracavity Bragg grating
}

\author{
David Bitauld, ${ }^{1}$ Isabelle Zaquine, ${ }^{1, *}$ Alain Maruani, ${ }^{1}$ and Robert Frey ${ }^{1,2}$ \\ 1GET/Laboratoire Traitement et Communication de l'Information, École Nationale Supérieure des Télécommunications, \\ 46, rue Barrault, F75634 Paris cedex 13, France \\ 'Laboratoire Charles Fabry de l'Institut d'Optique, Centre Scientifique d'Orsay Bât 503, F91403 Orsay cedex, France \\ ${ }^{*}$ Corresponding author: isabelle.zaquine@enst.fr
}

Received 13 November 2006; revised 8 February 2007; accepted 26 March 2007;

posted 27 March 2007 (Doc. ID 76991); published 6 July 2007

\begin{abstract}
A fast tunable filtering technique is proposed associating a diffraction grating with an intracavity Bragg grating. The bandwidth and the tuning range of this filter can be easily adapted by changing the diffraction grating's orientation, or its period, and its response is uniform over the whole tuning range. A numerical simulation of the filter response to a Gaussian beam has been developed, and it fits the experimental results allowing a calculation of the performances that could be obtained with more specific elements. For example, using a commercial acousto-optic deflector would allow a separation of 500 frequencies. It would then be possible to have a tuning range of $100 \mathrm{~nm}$ with a bandwidth of $0.2 \mathrm{~nm}$ for optical telecommunications. (C) 2007 Optical Society of America
\end{abstract}

OCIS codes: $\quad 060.0060,230.1040,350.2460,300.6170,120.2440$.

\section{Introduction}

The exponentially growing need for information transmission requires telecommunication networks with increasing rates combined with a high fluidity of the routing. While the transmission of the data is carried out optically through fibers, the steering has traditionally been executed by electronics. However, the latter reaches its limits as far as rate is concerned. A way to circumvent this problem is the multiplication of the channel number in a single fiber. This is implemented by modulating carrier waves of different wavelengths, a technique called wavelength division multiplexing (WDM). The densification of the channels then implies that the wavelengths of the carriers get very close: typically $0.2 \mathrm{~nm}$ in dense wavelength multiplexing (DWDM). To steer the information carried by each channel individually, one needs devices able to single out very accurately one wavelength. This operation is physically executed by tunable optical filters [1], add-drop filters [2], or wavelength switches [3-6]. These devices have to switch from one wavelength to another very quickly to match the continuous changes

0003-6935/07/214728-08\$15.00/0

(C) 2007 Optical Society of America in the network traffic. Tunable filters are the subject of intensive research and a wide variety of techniques are studied: tunable Bragg gratings (often in acousto-optic (AO) cells using surface waves [7-9] but also in fibers [10-13]), tunable Fabry-Perot [14-19] and diffraction gratings associated to steering devices like micromirrors [20-22] or deflectors [23,24].

The technique proposed in this paper combines the high dispersion of traditional diffraction gratings with the angular selectivity of intracavity Bragg gratings (IBG) demonstrated by Menez et al. [25]. In fact, the angular selectivity of the IBG is converted into wavelength selectivity through the angular dispersion of the diffraction grating. The performance of this filtering technique has been demonstrated experimentally using an intracavity liquid crystal grating [26] and an AO grating [27]. The AO tuning of the filter makes it faster (microsecond range) than most of the other techniques previously mentionned. In this paper, a numerical analysis developed to simulate our filtering principle is presented. These computations are successfully compared with the experimental results and then used to simulate optimized filters for specific applications.

In Section 2, we explain the mechanism of the filter. In Section 3, the details are given of the equations used 
in our model. In Section 4, we validate the simulation by comparing its results with the experimental data. The computed characteristics of an optimized filter are finally presented in Section 5.

\section{Principle}

The polychromatic light guided by the fiber (Fig. 1) is collimated by an objective into a Gaussian beam. It is dispersed by the diffraction grating $G$ so that the incidence angle on the intracavity Bragg grating depends on the wavelength. The particular construction of the IBG $[25,28]$ with its grating fringes perpendicular to the mirrors of the asymmetric cavity $\left(R_{1}\right.$ $\neq 1$ and $R_{2} \simeq 1$ ) implies that only the light arriving at the Bragg angle is efficiently diffracted (the cavity being tuned to this incidence), counterpropagating the incident beam. The light arriving at a different incidence is reflected by the IBG. The diffracted beam can easily be retrieved using a beam splitter or a circulator [29]. In that way, the light corresponding to a particular wavelength is separated from the rest of the polychromatic beam. To switch from this wavelength to another, one can change the grating period so that the Bragg condition is fulfilled for the new wavelength, and the corresponding beam returns in counterpropagation. In practice, the IBG diffracts the light in a certain range of incidence, but only the light having exactly the Bragg incidence is returned in precise counterpropagation. The filter can be made more selective through spatial filtering $[26,27]$. This can be implemented, for instance, by the injection of the diffracted beam into a single-mode fiber (see Fig. 1).

Simple expressions of the selectivity $\delta \lambda$ and tuning range $\Delta \lambda$ of the filter can be obtained when the filter selectivity is limited by the final spatial filtering (as is the case of the experiments described in $[26,27]$ for instance). Since the diffraction grating period is much smaller than the acoustic period $\Lambda$, the wavelength dispersion of the IBG grating can be neglected compared with that of the diffraction grating, and the spectral bandwidth is

$$
\delta \lambda=\lambda / 2 N_{R},
$$

where $N_{R}$ is the number of grooves of the diffraction grating that are illuminated by the beam. $N_{R}$ depends

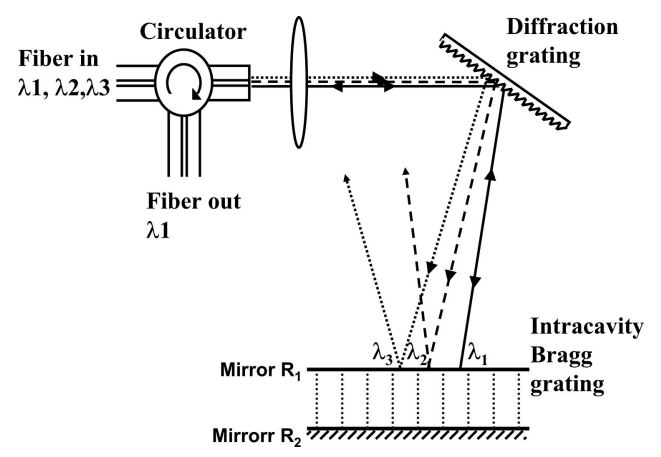

Fig. 1. Filtering principle. on the ratio of the beam diameter to the diffraction grating period. The tuning range is

$$
\Delta \lambda=\frac{\lambda}{2} \frac{\Delta \Lambda}{\Lambda} \frac{N_{B}}{N_{R}},
$$

where $\Delta \Lambda$ is the Bragg grating tuning range, determined by the $\mathrm{AO}$ device, and $N_{B}$ is the number of periods of the IBG illuminated by the beam. It can be noted that the diameter of the beam incident on the IBG depends on the dispersion of the diffraction grating. The Fabry-Perot etalon is adjusted for a maximum transmission at the desired wavelength to provide a great enhancement of the filter efficiency, but it does not influence the tuning range of the filter. The number of resolvable wavelengths is then

$$
N=\frac{\Delta \Lambda}{\Lambda} N_{B} .
$$

Since its spectral bandwidth essentially depends on $N_{R}$ and the number of resolvable lines depends on $N_{B}$ it is easy to optimize them separately.

These simple equations give a good idea of the filter selectivity that can be reached. Nevertheless, to obtain information about the diffraction efficiency and also an accurate value of the transmission bandwidth, it is necessary to develop a numerical simulation taking into account all the aspects of the light propagation in the device.

\section{Model}

The aim of this model is to calculate the transmission $P_{\text {out }} / P_{\text {in }}$ of the filter as a function of the wavelength of the incoming light. For that purpose, we take into account the effect of each element on the propagation of the light beam. Part of this task has been carried out in a previous study, where a calculation of the diffraction of Gaussian beams in the IBG has been developed [28]. In this calculation, the Gaussian beam is expanded in a plane wave basis. An analytical expression of the diffraction efficiency of each plane wave is obtained through a coupled wave analysis [30], and the boundary conditions are imposed by the cavity. Those efficiencies are then numerically summed to provide the diffraction efficiency of the complete beam.

In the present work, the beam traveling in the filter is also expanded in plane waves. Note that all optical elements on the beam path are considered large compared with the beam waist. The deviation of each wave by the diffraction grating is considered to determine analytically the amplitude of the incident waves on the IBG surface (see Subsection 3.A and Appendix A). The amplitude of the diffracted reflected waves in the reference frame $\mathscr{R}(0, x, y, z)$ of the IBG is derived from the IBG diffraction calculation. Taking into account the deviation attributable to the diffraction grating on the way back (Subsection 3.B and Appendix B), an analytical value of the incident field on the spatial filter is obtained. A numer- 


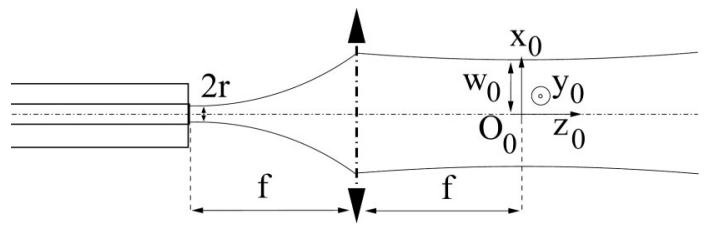

Fig. 2. Collimation of the beam from the fiber.

ical sum is then carried out to determine the power of the light going through the spatial filter.

\section{A. Way to the Intracavity Bragg Gratings}

In our filter, the incoming beam is collimated from a single-mode fiber. The fiber mode of radius $r$ is collimated by an objective of focal length $f$ into a beam of waist $w_{0}=f \lambda / \pi r$ (Fig. 2). In the reference frame of the objective $\mathscr{R}_{0}\left(O_{0}, x_{0}, y_{0}, z_{0}\right)$, the complex electric field of the incident light is described as a plane wave of wave vector $\mathbf{k}_{0}^{(0)}(0,0, k)$ multiplied by an amplitude $R_{0}\left(x_{0}, y_{0}\right)$ that defines the Gaussian shape of the beam. The amplitude $\widetilde{R_{0}}\left(\delta k_{x_{0}}, \delta k_{y_{0}}\right)$ of a Fourier component of $R_{0}\left(x_{0}, y_{0}\right)$ is then

$\widetilde{R_{0}}\left(\delta k_{x_{0}}, \delta k_{y_{0}}, z_{0}\right)=\sqrt{\frac{2 P_{i n} w_{0}^{2}}{c}} \exp \left[-\frac{w_{0}^{2}}{2}\left(\delta k_{x_{0}}{ }^{2}+\delta k_{y_{0}}{ }^{2}\right)\right]$,

where $P_{i n}$ is the power incident onto the filter. Note that this expression is valid because we are interested in the propagation of a low-divergence beam on a small length.

The input beam is thus expanded into plane waves whose wave vectors can be written as the wave vector of the central plane wave $\mathbf{k}_{\mathbf{0}}{ }^{(\mathbf{0})}$ to which a deviation $\delta \mathbf{k}_{\mathbf{0}}=\left(\delta k_{x_{0}}, \delta k_{y_{0}}, \delta k_{z_{0}}\right)$ is added. The changes undergone by the plane wave components at each element of the filter are explained in Appendix A.

It is therefore possible to derive the Fourier component amplitude $\widetilde{R_{I}}\left(\delta k_{x}, \delta k_{y}\right)$ of the beam incident onto the IBG, expressed in the frame $\mathscr{R}$ :

$$
\begin{aligned}
\widetilde{R_{I}}\left(\delta k_{x}, \delta k_{y}\right)= & \sqrt{\frac{2 P_{I} w_{0}{ }^{2}}{c n_{0}}} \exp \left[-\frac{w_{0}{ }^{2}}{2}\left(-\frac{\cos \left(i_{1}\right)}{\left(\cos \left(i_{0}\right) \cos \left(\theta_{e}\right)\right)}\right.\right. \\
& \left.\left.\times \delta k_{x}\right)^{2}+\delta k_{y}{ }^{2}\right] .
\end{aligned}
$$

Reference [28] is used to derive the Fourier components amplitudes of the forward and backward read and diffracted beams propagating in the IBG. The Fourier components amplitudes of the reflecteddiffracted beam coming out of the IBG $\tilde{S}_{D R}\left(\delta k_{x}, \delta k_{y}, 0\right)$ can then be computed and used for the analysis of the propagation to the output of the filter.

\section{B. Way Back from the Intracavity Bragg Gratings}

The amplitude $\tilde{S}_{0}\left(k_{3_{x_{0}}}, k_{3_{y_{0}}}\right)$ of the Fourier component of the field entering the spatial filter is given by
$\widetilde{S_{D R}}\left(\delta k_{x}, \delta k_{y}\right)$ provided the path of the reflecteddiffracted beam from the IBG to the output spatial filter is taken into account. This is detailed in Appen$\operatorname{dix} B$.

The total power $P_{\text {out }}$ coming out of the filter that can be coupled to the fundamental mode of a fiber is given by [31]

$$
P_{\text {out }}=\frac{c}{2 \pi} \frac{\left|\iint_{-\infty}^{+\infty} \mathrm{d} x_{F} \mathrm{~d} y_{F} E_{F}^{*}\left(x_{F}, y_{F}\right) E_{M}\left(x_{F}, y_{F}\right)\right|^{2}}{\iint \mathrm{d} x_{F} \mathrm{~d} y_{F}\left|E_{M}\left(x_{F}, y_{F}\right)\right|^{2}},
$$

where the amplitude of the actual field at the surface of the fiber (the filter output) $E_{F}\left(x_{F}, y_{F}\right)$ is projected on the Gaussian modal field amplitude of this fiber $E_{M}\left(x_{F}, y_{F}\right)$. It can be noted that the Gaussian mode is normalized

$$
\iint \mathrm{d} x_{F} \mathrm{~d} y_{F}\left|E_{M}\left(x_{F}, y_{F}\right)\right|^{2}=1 \text {. }
$$

The objective of the spatial filter performing a Fourier transform on the field, in the paraxial approximation used in this analysis, the power $P_{\text {out }}$ can also be obtained by summing on the Fourier transform of the field incident on the objective:

$P_{\text {out }}=\frac{c}{2 \pi}\left|\iint_{-\infty}^{+\infty} \mathrm{d} k_{3_{x_{0}}} \mathrm{~d} k_{3_{y_{0}}} \tilde{S}_{0}\left(k_{3_{x_{0}}}, k_{3_{y_{0}}}\right) \tilde{M}\left(k_{3_{x_{0}}}, k_{3_{y_{0}}}\right)\right|^{2}$,

where $\tilde{S}_{0}\left(k_{3_{x_{0}}}, k_{3_{y_{0}}}\right)$ and $\tilde{M}\left(k_{3_{x_{0}}}, k_{3_{y_{0}}}\right)=w_{0} / \sqrt{\pi} \exp [-$ $\left.\left(w_{0}^{2} / 2\right)\left(k_{3_{x_{0}}}{ }^{2}+k_{3_{y_{0}}}\right)\right]$ are the Fourier transforms of $E_{F}^{*}\left(x_{F}, y_{F}\right)$ and $E_{M}\left(x_{F}, y_{F}\right)$ respectively. Note that for a beam coming out of the filter (wave vector $\mathbf{k}_{3}$, see Fig. $3)$, as $E_{F} *\left(x_{F}, y_{F}\right)$ and $E_{M}\left(x_{F}, y_{F}\right)$ are the fields in the image focal plane of the objective used for the spatial filtering at the output, $\tilde{S}_{0}\left(k_{3_{x_{0}}}, k_{3_{y_{0}}}\right)$ and $\tilde{M}\left(k_{3_{x_{0}}}, k_{3_{y_{0}}}\right)$ are the Fourier transforms of these fields in the object focal plane. Moreover, we use the fact that, in the paraxial approximation, $\tilde{S}_{0}\left(k_{3_{x_{0}}}, k_{3_{y_{0}}}\right)$ and $\tilde{M}\left(k_{3_{x_{0}}}, k_{3_{y_{0}}}\right)$ also represent the field distributions in the lens plane

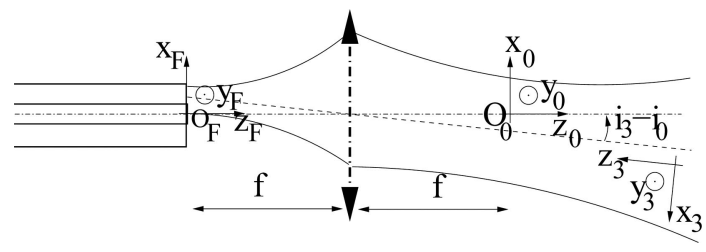

Fig. 3. Reinjection of the light in a fiber. 
within a constant phase change. We found out that it was easier to perform the numerical sum in the $\mathscr{B}_{3}$ basis (see Appendix B). After the adequate variable change, the power coupled into the fiber is then given by

$$
\begin{aligned}
P_{\text {out }}= & \frac{c}{2 \pi} \mid \iint_{-\infty}^{+\infty}\left(\frac{\cos \left(i_{2}\right) \cos \left(i_{3}-i_{0}\right)}{\cos \left(i_{3}\right) \cos \left(\theta_{e}^{\prime}\right)} \mathrm{d} \delta k_{x} \mathrm{~d} \delta k_{y} \tilde{S}_{D R}\left(\delta k_{x}, \delta k_{y}, 0\right)\right) \\
& \times\left. M\left(\frac{\cos \left(i_{2}\right) \cos \left(i_{3}-i_{0}\right)}{\cos \left(i_{3}\right) \cos \left(\theta_{e}^{\prime}\right)} \delta k_{x}-k \sin \left(i_{3}-i_{0}\right), \delta k_{y}, 0\right)\right|^{2} .
\end{aligned}
$$

When the spatial filtering is performed by a hole, as in the experiments described in [27] Eq. (9) is no longer valid. The transmitted power is then

$$
P=\frac{c}{2 \pi} \iint_{x_{F}^{2}+y_{F}^{2}<r^{2}} \mathrm{~d} x_{F} \mathrm{~d} y_{F}\left|E_{F}\left(x_{F}, y_{F}, 0\right)\right|^{2} .
$$

In this case, the transmitted power is calculated from the integral over the plane of the hole $\left(x_{F}{ }^{2}+y_{F}{ }^{2}\right.$ $\left.<r^{2}\right)$ of the intensities of $E_{F}\left(x_{F}, y_{F}, 0\right)$. This condition can also be expressed in the Fourier space, either by using the Parseval theorem or by noting that only the plane waves converging on the points $\left(x_{F}, y_{F}, 0\right)$ satisfying $x_{F}^{2}+y_{F}^{2}<r^{2}$ go through the hole. This last inequality is equivalent to

$$
\left(\frac{k_{3_{x_{0}}} f}{k}\right)^{2}+\left(\frac{k_{3_{y_{0}}} f}{k}\right)^{2}<r^{2}
$$

and it is therefore convenient to use polar coordinates in the Fourier space: $k_{3_{x_{0}}}=\rho \cos \phi$ and $k_{3_{y_{0}}}$ $=\rho \sin \phi$. The light power that is transmitted is given by the sum of the squared modulus of amplitudes of the plane waves fulfilling the latter condition. The power injected into the hole is therefore

$$
P=\frac{c}{2 \pi} \int_{0}^{2 \pi} \mathrm{d} \phi \int_{0}^{r k / f} \rho \mathrm{d} \rho\left|\tilde{S}_{0}(\rho \cos \phi, \rho \sin \phi, 0)\right|^{2},
$$

or

$$
P=\frac{c}{2 \pi} \int_{0}^{2 \pi} \mathrm{d} \phi \int_{0}^{r k / f} \rho \mathrm{d} \rho\left|\tilde{S}_{D R}\left(\delta k_{x}, \delta k_{y}, 0\right)\right|^{2}
$$

with

$$
\begin{aligned}
& \delta k_{x}=\left(\rho \cos \phi-\left(\frac{2 \pi}{\lambda_{0}}\right) \sin \left(i_{3}-i_{0}\right)\right) \frac{\cos \theta_{e}{ }^{\prime} \cos i_{3}}{\cos \left(i_{3}-i_{0}\right) \cos i_{2}}, \\
& \delta k_{y}=\rho \sin \phi .
\end{aligned}
$$

The simulation of the filter's response is then complete, and its results can be compared with the experiments.

\section{Numerical Analysis of Experimental Data at $\mathbf{7 6 0} \mathbf{n m}$}

In the experiments described in [27], a commercial AO cell, which is actually a modulator, is used with a mirror at its back. As the acoustic velocity in this $3 \mathrm{~mm}$ wide $\mathrm{TeO}_{2}$ crystal is $4200 \mathrm{~m} / \mathrm{s}$, it takes less than $1 \mu \mathrm{s}\left(3.10^{-3} / 4200=0.7 \times 10^{-7} \mathrm{~s}\right)$ to change the acoustic frequency, in order to tune the filter to a new wavelength. This configuration is equivalent to an intracavity Bragg grating with a null reflectivity for the front mirror. It is thus not angularly very selective, but the desired wavelength can be selected with a good resolution through spatial filtering. The returning light is extracted with a beam splitter and injected, thanks to a lens, into a pinhole, which achieves the spatial filtering. The light source is a tunable Ti-Sa laser operated in the single-mode regime, using a fixed intracavity Fabry-Perot etalon. As a consequence, the wavelength is not tuned continuously but jumps discretely from one mode of the etalon to the other with a wavelength step of $0.43 \mathrm{~nm}$. To obtain a Gaussian beam, the laser light is injected into a single-mode fiber and collimated in a $0.5 \mathrm{~mm}$ waist beam. The diffraction grating has a $555 \mathrm{~nm}$ period and is used in the Littman configuration. The
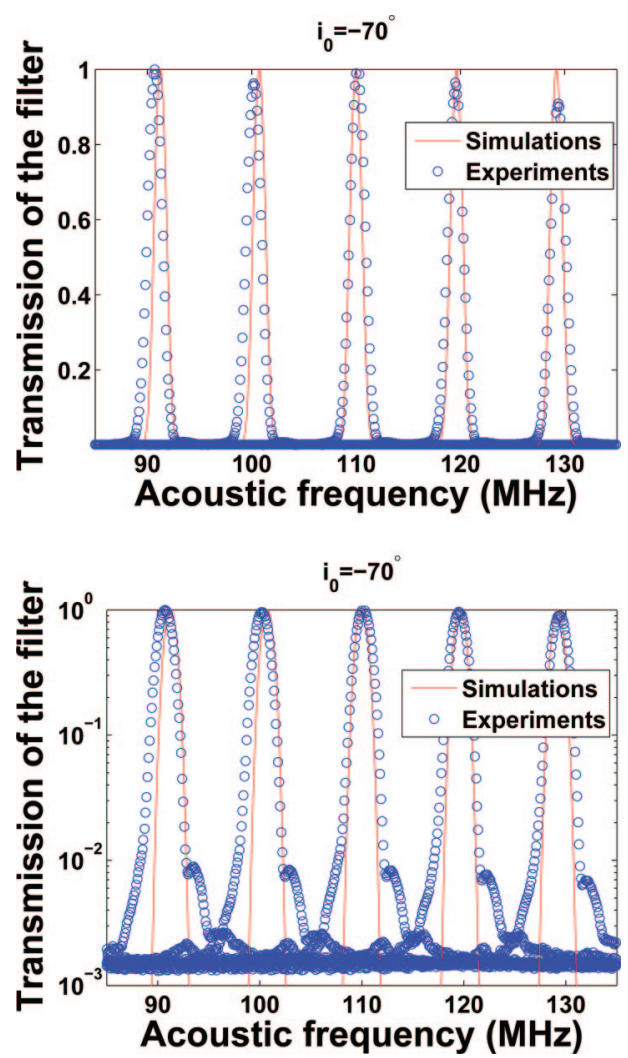

Fig. 4. Transmission of the filter as a function of the acoustic frequency for five wavelengths at $\sim 760 \mathrm{~nm}$ with a space of $0.43 \mathrm{~nm}$ ( $225 \mathrm{GHz}$ exactly). Linear scale on the upper curve and semilogarithmic scale on the lower one. 
beam is incident at $70^{\circ}$ on the diffraction grating. For five different $\mathrm{Ti}-\mathrm{Sa}$ laser wavelengths at approximately $760 \mathrm{~nm}$, the transmission of the filter is measured as a function of the acoustic frequency, the latter being directly linked to the $\mathrm{AO}$ grating period. The corresponding spectra are shown in Fig. 4. The acoustic FWHM is $1.6 \mathrm{MHz}$, which gives the possibility to resolve 30 different optical frequencies within the $50 \mathrm{MHz}$ tuning range of the $\mathrm{AO}$ modulator used in our experiment. The linear scale shows the good uniformity of the filter transmission; the selected wavelength is always at Bragg incidence and experiences the same diffraction efficiency, in the whole tuning range, which would not be the case with other filtering techniques using AO devices [24]. On the logarithmic scale, one can see that the sidelobes are down to $20 \mathrm{~dB}$ below the maxima, which makes them quite harmless for most applications. They are due to the limited aperture of the AO cell, causing diffraction of the beam, as it is widened after the diffraction on the grating. The demonstrated optical tuning range is $2.2 \mathrm{~nm}$, centered on $760 \mathrm{~nm}$.

In Fig. 4, the experimental data can be compared with the simulations using precisely the experimental parameters. The curves are normalized because the losses on the diffraction grating and the beam splitter are not taken into account. Anyway, these losses could be made very small by using a circulator instead of the beam splitter as well as an adapted diffraction grating.

Moreover, although there is no adjustable parameter, a very good agreement between experiments and simulation is obtained on the position and width of the peaks, which provides a validation of our numerical simulation. It is then possible to calculate the performances of optimized filters.

\section{Optimized Filters at $\mathbf{1 5 5 0} \mathbf{n m}$}

The AO cell Bragg grating used in the experiments of [27] allowed us to test the feasibility of the filter and to validate the simulation. Nevertheless, no element of the device was specially designed for this application. We now present the calculated performances that could be reached with commercially available Bragg and diffraction gratings specifically chosen to optimize the tunable filter for DWDM communication. The impact of the wavelength range at approximately $1.55 \mu \mathrm{m}$ is taken into account in the equation, but the main change would come from the choice of an optimized AO cell and a suitable diffraction grating period, as explained hereafter.

The main limitation of the experimental filter presented in Section 4 is the width of the Bragg grating or more precisely the number $N_{R}$ of illuminated periods of the $\mathrm{AO}$ cell, as it is directly bound to the number of channels in the tuning range of the filter
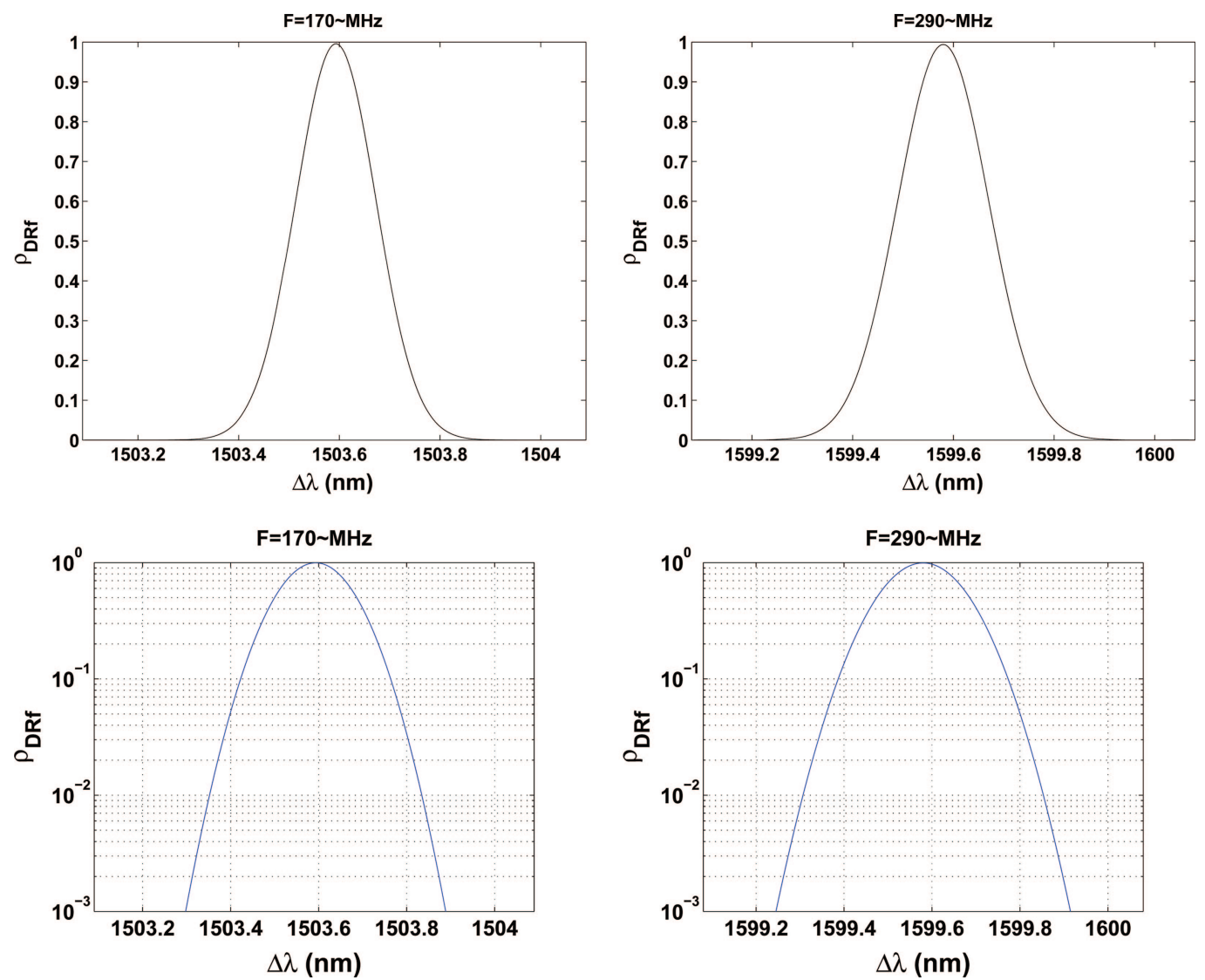

Fig. 5. Transmission spectrum of the filter at approximately $1550 \mathrm{~nm}$ with a $17 \mathrm{~mm}$ wide acousto-optic cell at an acoustic frequency of $170 \mathrm{MHz}$ (left) and $290 \mathrm{MHz}$ (right). The upper plots have a linear scale and the lower ones have a semilogarithmic scale. 
[see Eq. (3)]. Commercial AO deflectors can be found with a width of $17 \mathrm{~mm}$ (to be compared with the $3 \mathrm{~mm}$ width of our modulator). Moreover, their acoustic tuning range is $120 \mathrm{MHz}$ instead of $50 \mathrm{MHz}$ for the modulator. It is interesting to evaluate how these characteristics can improve the filter performances.

With the help of Eq. (1), a configuration is chosen that generates transmission peak widths of $0.2 \mathrm{~nm}$ to match the DWDM communications requirements. We choose a beam waist $w_{0}$ of $5 \mathrm{~mm}$ and an incidence angle $i_{0}=-40^{\circ}$ on a diffraction grating with a period $a=4 \mu \mathrm{m}$. The angle between the Bragg and the diffraction grating is chosen so that the tuning range spreads at approximately $1.55 \mu \mathrm{m}$.

Figure 5 shows the transmission peaks for acoustic frequencies of 170 and $290 \mathrm{MHz}$ that correspond to the limits of the tuning range spreading from 1503, $6 \mathrm{~nm}$ to $1599,6 \mathrm{~nm}(\Delta \lambda=96 \mathrm{~nm})$. The linear scale of the upper plots is convenient to see the channel bandwidth whereas the semilogarithmic scale of the lower plots is useful to evaluate the cross-talk performances. The FWHM of the peaks are $\delta \lambda=$ $0,19 \mathrm{~nm}(24 \mathrm{GHz})$ for $F=170 \mathrm{MHz}$ and $\delta \lambda=$ $0,21 \mathrm{~nm}(27 \mathrm{GHz})$ for $F=290 \mathrm{MHz}$. The number of distinguishable wavelengths is thus $N=480$. The widths of the peaks at $-25 \mathrm{~dB}$ are $0,55 \mathrm{~nm}(70 \mathrm{GHz})$ and $0,61 \mathrm{~nm}(76 \mathrm{GHz})$. The number of telecommunication channels with an overlap of $-25 \mathrm{~dB}$ is 165 , with a perfect transmission uniformity. The tuning speed would be $4 \mu \mathrm{s}$ in this $17 \mathrm{~mm}$ wide crystal. It can be concluded that this device is most interesting for DWDM communications.

Note that the add-drop function could be achieved with an angularly very selective IBG, obtained with a very high finesse Fabry-Perot resonator. As shown in Fig. 1 of [27] the reflected light would also be retrieved using another diffraction grating set symmetrically to the first one, to redirect all remaining wavelengths toward the same direction. The device could also be used as the external cavity of a tunable laser, especially in the case of a fiber laser where the reinjection into the fiber automatically achieves the necessary spatial filtering.

\section{Conclusion}

A high performance filtering technique associating a diffraction grating to an intracavity Bragg grating has been demonstrated. The developed simulation is in good agreement with the experimental results and is used to design optimized devices. A simple setup without a cavity and using commercially available elements could have a transmission bandwidth of $0.2 \mathrm{~nm}$ with a tuning range of $96 \mathrm{~nm}$, which makes it suitable for a DWDM application. The adaptability of this filtering technique is very wide. The selectivity and the tuning range can be chosen just by turning or changing the diffraction grating. The only limitation is the number of distinguishable frequencies, which is given by the characteristics of the Bragg grating (especially its width). Add-drop functions, bandpass filter and tunable lasers could also be achieved with a more sophisticated intracavity Bragg grating, which can be designed using the theoretical analysis given herebefore.

\section{Appendix A: Way to the Intracavity Bragg Gratings}

This is the detailed analysis of the propagation of the plane wave components of the Gaussian beam from the input of the filter to the IBG. The relation between the wave vectors deviation from the central propagation direction $\delta \mathbf{k}_{\mathbf{0}}=\left(\delta k_{x_{0}}, \delta k_{y_{0}}\right)$ and $\delta \mathbf{k}=$ $\left(\delta k_{x}, \delta k_{y}\right)$ of the plane wave components at the injection objective and the IBG, respectively, is determined by the beam propagation from the output of the fiber to the IBG. It then depends on the incidence angles $i_{0}$ and $\theta_{e}$ of the central wave vector on the traditional and intracavity gratings, respectively. $\theta_{e}$ depends on the angle between the gratings' normal $\alpha$ via the relation $\theta_{e}=i_{0}-\alpha$. These angles are represented in Fig. 6. For each part of the path, the wave vectors can be expressed in a basis whose $z$ axis is in the direction of the central wave vector. The relations between the directions of a given plane wave before and after an element are easily determined by writing their wave vectors in the basis of the element (all bases are represented in Figs. 6 and 7). As no direction change occurs along the $y$ axis, the deviation $\delta k_{y}$ is constant during the whole process and we only need to relate the $x$ deviation $\delta k_{x_{i}}$ to $\delta k_{x_{i+1}}$. This is done by applying the conservation of the wave-vector projection on the boundary plane, taking its periodicity into account. The incoming wave vector $\mathbf{k}_{\text {in }}$ is related to the outcoming one $\mathbf{k}_{\text {out }}$ in the following way:

$$
\mathbf{k}_{\text {in }} \cdot \hat{\mathbf{x}}-\mathbf{k}_{\text {out }} \cdot \hat{\mathbf{x}}=0
$$

for refraction in the IBG medium, and

$$
\left(\mathbf{k}_{\text {in }} \pm \mathbf{K}\right) \cdot \hat{\mathbf{x}}_{\mathbf{j}}-\mathbf{k}_{\text {out }} \cdot \hat{\mathbf{x}}_{\mathbf{j}}=0
$$

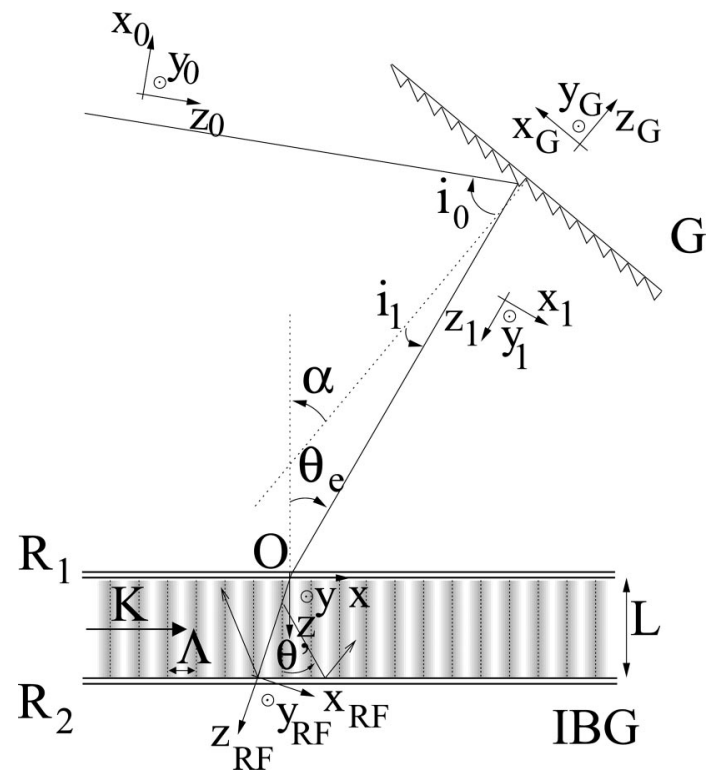

Fig. 6. (Color online) Angles defining the path of the central plane wave from the fiber to the IBG. 


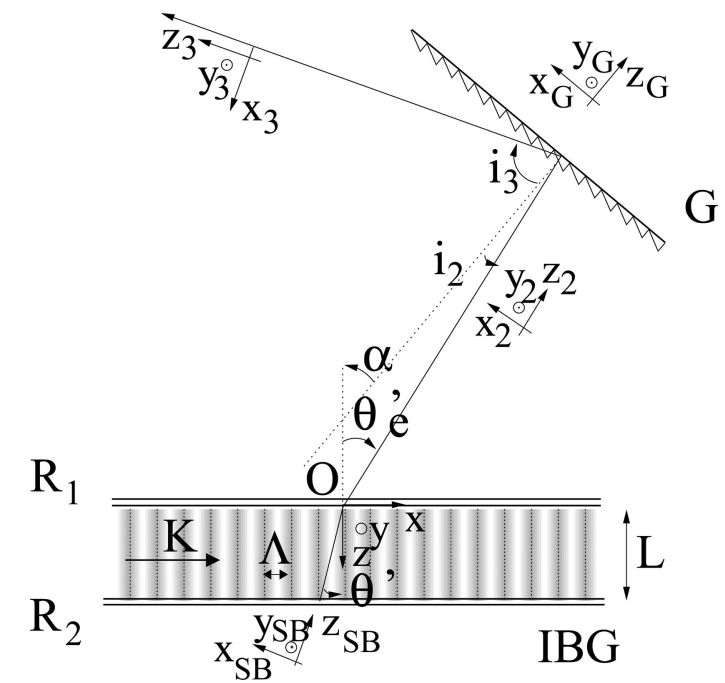

Fig. 7. Angles defining the path of the central plane wave from the IBG back to the fiber.

for diffraction by a grating with a wavevector $\mathbf{K}$ parallel to $\hat{\mathbf{x}}_{\mathbf{j}}$.

Table 1 shows the relations between the incidence angles of the central wave and also between the deviations of a given off-axis plane wave at each interface. $\mathbf{K}_{\mathbf{G}}$ is the wave vector of the grating $G$.

A deviation $\delta k_{x_{0}}$ with respect to the central wave vector at the fiber exit leads to a deviation $\delta k_{x_{R F}}$ at the surface of the IBG, and a relation between those deviations can be obtained by using Table 1 . Expressed in the reference frame $\mathscr{R}(O, x, y, z)$ the deviation becomes $\delta k_{x}=\delta k_{x_{R F}} \cos (\theta)$. We have thus the relation

$$
\delta k_{x_{0}}=-\frac{\cos \left(i_{1}\right)}{\cos \left(i_{0}\right) \cos \left(\theta_{e}\right)} \delta k_{x}
$$

and $\delta k_{y_{0}}=\delta k_{y}$.

\section{Appendix B: Way Back from the Intracavity Bragg Gratings}

This is a detailed analysis of the propagation of the plane wave components of the Gaussian beam from the IBG to the output of the filter. Therefore we need to express the central wave vectors $\mathbf{k}_{\mathbf{i}}$ and the wave vectors of the plane wave components $\delta \mathbf{k}_{\mathbf{i}}$ of the beam at every stage of the path so that $\mathbf{k}_{\mathbf{3}}$ and $\delta \mathbf{k}_{\mathbf{3}}$ $=\left(\delta k_{3_{x_{0}}}, \delta k_{3_{y_{0}}}\right)$ at the output of the filter in the reference frame of the output objective can be related to $\mathbf{k}$ and $\delta \mathbf{k}=\left(\delta k_{x}, \delta k_{y}\right)$ in the IBG.

Table 1. Relations Between Wave Vectors Before the Intracavity Bragg Gratings

\begin{tabular}{ccc}
\hline & Diffraction & Refraction \\
\hline $\begin{array}{c}\text { Central } \\
\text { wave }\end{array}$ & $i_{1}=-\arcsin \left(\sin \left(i_{0}\right) \pm \frac{K_{G}}{k}\right)$ & $\theta=\arcsin \left(\frac{\sin \left(\theta_{e}\right)}{n}\right)$ \\
$\begin{aligned} \text { Off-axis } \\
\text { plane }\end{aligned}$ & $\delta k_{x_{1}}=-\frac{\cos \left(i_{0}\right)}{\cos \left(i_{1}\right)} \delta k_{x_{0}}$ & $\delta k_{x_{R F}}=\frac{\cos \left(\theta_{e}\right)}{\cos (\theta)} \delta k_{x_{1}}$ \\
wave & & \\
\hline
\end{tabular}

Table 2. Relations Between Wave Vectors After the Intracavity Bragg Gratings

\begin{tabular}{ccc}
\hline & Refraction & Diffraction \\
\hline $\begin{array}{c}\text { Central } \\
\text { wave }\end{array}$ & $\theta_{e}{ }^{\prime}=\arcsin \left(n \sin \left(\theta^{\prime}\right)\right)$ & $i_{3}=-\arcsin \left(\sin \left(i_{2}\right) \pm \frac{K_{G}}{k}\right)$ \\
$\begin{array}{c}\text { Off-axis } \\
\text { plane } \\
\text { wave }\end{array}$ & $\delta k_{x_{2}}=\frac{\cos \left(\theta^{\prime}\right)}{\cos \left(\theta_{e}{ }^{\prime}\right)} \delta k_{x_{S B}}$ & $\delta k_{x_{3}}=-\frac{\cos \left(i_{2}\right)}{\cos \left(i_{3}\right)} \delta k_{x_{2}}$ \\
\hline
\end{tabular}

As in the case of the forward propagation in the filter, this relation depends on the incidence angles $\theta$ and $\theta_{2}$ and emergence angles $\theta_{e}{ }^{\prime}$ and $\theta_{3}$ of the central wave vector on $G$ and IBG (see Fig. 7). Table 2 shows the relations between the incidence angles of the central wave and also between the deviations of a given off-axis plane wave at each stage. These relations are obtained by using Eqs. (A1) and (A2) for the way to the output of the filter. From Table 2 and using $\delta k_{x_{S R}} \cos \theta^{\prime}=\delta k_{x}$ in order to take the tilt of the IBG into account, we get

$$
\delta k_{x_{3}}=-\frac{\cos \left(i_{2}\right)}{\cos \left(\theta_{e}{ }^{\prime}\right) \cos \left(i_{3}\right)} \delta k_{x}
$$

in the frame $\mathscr{B}_{3}\left(O_{3}, x_{3}, y_{3}, z_{3}\right)$ corresponding to the beam at the entrance of the spatial filter. In the focal plane of the objective $\left(O_{0}, x_{0}, y_{0}\right)$ the central wave vector of the incoming beam $\mathbf{k}_{\mathbf{3}}{ }^{(\mathbf{0})}$ is at an angle $i_{3}$ $i_{0}+\pi$ to the original beam. Only a monochromatic beam having exactly the selected wavelength is in precise contrapropagation and thus at an angle of $\pi$. The final step is to change the basis from $\mathscr{B}_{3}$ to the basis of the objective $\mathscr{B}_{0}$ through a $\pi+i_{3}-i_{0}$ rotation about $y_{3}$. Using the equations of Tables 1 and 2 , it is then possible to express the vector $\mathbf{k}_{\mathbf{3}}\left(k_{3_{x_{2}}}, k_{3_{y_{0}}}, k_{3_{z_{0}}}\right)$ as a function of the variables $\delta k_{x}$ and $\delta k_{y}$, which are used in the IBG diffraction model conputation. We thus have

$$
k_{x_{3}}=\frac{\cos \left(i_{2}\right)}{\cos \left(i_{3}\right) \cos \left(\theta_{e}{ }^{\prime}\right)} \delta k_{x} \cos \left(i_{3}-i_{0}\right)+k \sin \left(i_{3}-i_{0}\right) .
$$

\section{References}

1. D. Sadot and E. Boimovich, "Tunable optical filters for dense wdm networks," IEEE Commun. Mag. 36, 50-55 (1998).

2. M. Fukutoku, K. Oda, and H. Toba, "Wavelength-divisionmultiplexing add/drop multiplexer employing a novel polarisation independent acousto-optic tunable filter," Electron. Lett. 29, 905-907 (1993).

3. Y. W. Song, Z. Pan, D. Starodubov, V. Grubsky, E. Salik, S. A. Havstad, Y. Xie, A. E. Willner, and J. Feinberg, "All-fiber wdm optical crossconnect using ultrastrong widely tunable fbgs," IEEE Photon. Technol. Lett. 13, 1103-1105 (2001).

4. J. E. Ford, V. A. Aksyuk, D. J. Bishop, and J. A. Walker, "Wavelength add drop switching using tilting micromirrors," J Lightwave Technol. 17, 904-911 (1999).

5. D. Marom, D. Neilsont, and D. Greywall, "Wavelengthselective $1 \times 4$ switch for 128 wdm channels at 50 ghz spacing," in Optical Fiber Communication Conference and Exhibit, 2002 (OFC, 2002), pp. FB7-1-FB7-3. 
6. W. Duncan, T. Bartlett, B. Lee, D. Powell, P. Rancuret, and B. Sawyers, "Dynamic optical filtering in dwdm systems using the dmd," Solid-State Electron. 46, 1583-1585 (2002).

7. B. Heffner, D. Smith, I. Baran, A. YI-Yan, and K. Cheung, "Integrated-optic acoustically tunable infrared optical filter," Electron. Lett. 24, 1562-1563 (1988).

8. K. W. Cheung, D. A. Smith, J. E. Baran, and B. L. Heffner, "Multiple channel operation of an integrated acousto-optic," Electron. Lett. 25, 375-376 (1989).

9. M. S. Borella, "Optical components for wdm lightwave networks," Proc. IEEE 85, 1274-1307 (1997).

10. D. Ostling and H. Engan, "Spectral flattening by an all-fiber acousto-optic tunable filter," in Proceeding of Ultrasonics Symposium (Seattle, 1995), Vol. 2, pp. 837-840.

11. H. S. Kim, S. H. Yun, I. K. Kwang, and B. Y. Kim, "Low-loss all-fiber acousto-optic tunable filter," Opt. Lett. 22, 1476-1478 (1997).

12. S. H. Yun, D. J. Richardson, D. O. Culverhouse, and T. A. Birks, "All-fiber acoustooptic filter with low-polarization sensitivity and no frequency shift," IEEE Photon. Technol. Lett. 9, 461-463 (1997).

13. D. S. Starodubov, V. Grubsky, and J. Feinberg, "All-fiber bandpass filter with adjustable transmission using cladding-mode coupling," IEEE Photon. Technol. Lett. 10, 1590-1592 (1998).

14. A. Spisser, R. Ledantec, C. Seassal, J. L. Leclercq, T. Benyattou, D. Rondi, R. Blondeau, G. Guillot, and P. Viktorovitch, "Highly selective and widely tunable $1.55 \mu \mathrm{m}$ inp/air-gap micromachined Fabry-Perot filter for optical communications," IEEE Photon. Technol. Lett. 10, 1259-1261 (1998).

15. R. Le Dantec, T. Benyattou, G. Guillot, A. Spisser, J. L. Leclercq, P. Viktorovitch, D. Rondi, and R. Blondeau, "Tunable microcavity based on inp air Bragg mirrors," IEEE J. Sel. Top. Quantum Electron. 5, 111-114 (1999).

16. P. Tayebati, P. D. Wang, D. Vakhshoori, and R. N. Sacks, "Widely tunable Fabry-Perot filter using ga(al)as alox deformable mirrors," IEEE Photon. Technol. Lett. 10, 394-396 (1998).

17. J. Peerlings, A. Dehe, A. Vogt, M. Tilsch, C. Hebeler, F. J. Langenhan, P. Meissner, and H. L. Hartnagel, "Long resonator micromachined tunable gaas alas Fabry-Perot filter," IEEE Photon. Technol. Lett. 9, 1235-1237 (1997).

18. J. Daleiden, V. Rangelov, S. Inner, E. Romer, M. Strassner, C. Prott, A. Tarraf, and H. Hillmer, "Record tuning range of inp-based multiple air-gap moems filter," Electron. Lett. 38, 1270-1271 (2002).

19. M. Strassner, J. C. Esnault, L. Leroy, J.-L. Leclercq, M. Gar- rigues, and I. Sagnes, "Fabrication of ultrathin and highly flexible inp-based membranes for micro-optoelectromechanical systems at $1.55 \mu \mathrm{m}$," IEEE Photon. Technol. Lett. 17, 804-806 (2005).

20. J. Berger, F. Ilkov, D. King, A. Tselikov, and D. Anthon, "Widely tunable, narrow optical bandpass Gaussian filter using a silicon microactuator," In Optical Fiber Communication Conference (OFC), Postconference Digest (IEEE, 2003), Vol. 86, pp. 252-253.

21. G. Wilson, C. J. Chen, P. Gooding, and J. E. Ford "Spectral filter with independently variable center wavelength and bandwidth," in 30th ECOC Proceedings, Stockholm, Sweden, September 2004.

22. W. Huang, R. R. A. Syms, J. Stagg, and A. Lohmann, "Precision mems flexure mount for a Littman tunable external cavity laser," IEE Proc.: Sci. Meas. Technol. 151, 67-75 (2004).

23. A. A. Tarasov, H. Chu, and Y. M. Jhon, "Polarizationindependent acousto-optically tuned spectral filter with frequency shift compensation," IEEE Photon. Technol. Lett. 14, 944-946 (2002)

24. E. G. Paek, J. Y. Choe, and T. K. Oh, "Transverse gratingassisted narrow-bandwidth acousto-optic tunable filter," Opt. Lett. 23, 1322-1324 (1998).

25. L. Menez, I. Zaquine, A. Maruani, and R. Frey, "Intracavity Bragg gratings," J. Opt. Soc. Am. B 16, 1849-1855 (1999).

26. D. Bitauld, C. Martins, I. Zaquine, A. Maruani, R. Frey, R. Chevallier, and L. Dupont, "Tunable optical filtering with an intracavity bragg grating associated to a standard grating," in Conference on Lasers and Electro-Optics, 2004 (CLEO) (IEEE, 2004), Vol. 2.

27. D. Bitauld, I. Zaquine, A. Maruani, and R. Frey, "Gratingassisted uniform response high resolution tunable optical filtering using a grating-assisted acousto-optic device," Opt. Express 13, 6438-6444 (2005).

28. D. Bitauld, L. Menez, I. Zaquine, A. Maruani, and R. Frey, "Diffraction of Gaussian beams on intracavity Bragg gratings," J. Opt. Soc. Am. B 22, 1153-1160 (2005).

29. Y. Fujii, "High-isolation polarization-independent optical circulator coupled with single-mode fibers," J. Lightwave Technol. 19, 1238-1243 (1991).

30. H. Kogelnik, "Coupled wave theory for thick hologram gratings,” Bell. Syst. Tech. J. 48, 2909-2947 (1969).

31. S. Vatoux, Y. Combemale, A. Enard, J. Arnoux, and M. Papuchon, L'optique Guide Monomode (Masson, 1985), pp. 663-710. 